Revista internacional de fronteras, territorios y regiones / International Journal of Borders, Territories and Regions

FRONTERA NORTE VOL. 32, ART. 8, 2020

http://dx.doi.org/10.33679/rfn.v1i1.1987

\title{
Emergencia y complejidad en los Sistemas Regionales de Innovación: aplicación de una metodología basada en agentes del sector biotecnológico del estado de Sonora
}

\section{Complexity and Emergence on Regional Innovation Systems: Applying an agent based methodology on the Sonoran biotech sector}

\author{
María Socorro Arvizu Arvizu ${ }^{1}$ y Álvaro Bracamonte Sierra ${ }^{2}$
}

\section{RESUMEN}

Este trabajo analiza los conceptos de emergencia y complejidad en el marco de un Sistema Regional de Innovación (SRI), en proceso de formación en el sector biotecnológico del estado de Sonora, México. Para ello, se explora la implementación de un sistema basado en agentes codificado en la plataforma NetLogo, con el fin de simular el comportamiento cooperativo en red de las firmas para el intercambio de conocimiento y el desarrollo de nuevos productos. El método es útil para la predicción y el análisis de posibles cursos de comportamiento en red de las firmas, y para el futuro desempeño del sistema. La incorporación y modulación de variables económicas relevantes para sistemas regionales en vías de formación dentro de sistemas no lineales constituye una herramienta poco explorada para el estudio de los SRI en países en desarrollo, por lo que con este artículo se espera alentar esta clase de investigaciones y contribuir a su mayor conocimiento.

Palabras clave: 1 . Análisis de redes sociales, 2. Modelos basados en agentes, 3. NetLogo, 4. Sistemas Regionales de Innovación, 5. Sonora.

\section{ABSTRACT}

Using a dataset gathered through a small sample of biotech firms in the Sonora region, we explore concepts of emergency and complexity inside a RIS (Regional Innovation System), in a development stage through the evolutionary microeconomics framework. For this, we explored the implementation of an ABS (Agent Based System) coded in the NetLogo platform, in which we simulated the behavior of firms in network behavior for the cooperation in the exchange of knowledge and new product development. We found this methodology highly useful for predictive analysis of possible courses of network behavior among different types of firms, as well as future performance of the system. Moreover, the coalescence and modulation of key economic variables for a RIS in development stages in nonlinear systems is an extraordinary and relatively unexplored tool for the study of Regional Innovation Systems (RIS) in developing countries.

Keywords: 1. Social Network Analysis, 2. Agent-Based Modeling, 3. NetLogo, 4. Regional Innovation Systems, 5. Sonora.

Fecha de recepción: 21 de marzo de 2019

Fecha de aceptación: 12 de julio de 2019

Publicación en web: 2 de julio de 2020

${ }^{1}$ El Colegio de Sonora, México, leia.y2k@gmail.com, https://orcid.org/0000-0002-3833-305X

${ }^{2}$ El Colegio de Sonora, México, abraca@colson.edu.mx, https://orcid.org/0000-0002-8523-0846 
2 Emergencia y complejidad en los Sistemas Regionales de Innovación...

Arvizu Arvizu, M. A. y Bracamonte Sierra, Á.

\section{INTRODUCCIÓN}

A pesar de ser la cuna de la llamada "Revolución verde", el estado de Sonora, ubicado en el noroeste de México, permanece rezagado en términos de innovación biotecnológica. ${ }^{3}$ No obstante, en dicha región reside una vibrante comunidad académica (investigadores, estudiantes de distintos niveles y al menos, tres instituciones y centros de investigación) que trabaja en el sector biotecnológico. Esta masa crítica constituye una sólida base de capital humano especializado, lo que sugiere la pertinencia de indagar en fenómenos de emergencia al interior de esta red.

La debilidad de esa red emergente se advierte en la escasa presencia de startups en el ramo, al igual que en una pobre generación de patentes, pese a la existencia de un nutrido flujo de proyectos de investigación provenientes de instituciones y centros de investigación que no logran cristalizar en una firma.

Dichas instituciones, que emergieron a raíz de la expansión agrícola, ganadera y pesquera en el estado, entre ellas la Universidad de Sonora (Unison), el Instituto Tecnológico de Sonora (Itson) y el Centro de Investigación en Alimentación y Desarrollo (CIAD), conjuntan más de 400 profesores adscritos al Sistema Nacional de Investigadores, y más de 100000 estudiantes de posgrado y licenciatura. Tan solo la Universidad de Sonora forma parte de comunidades científicas de k-núcleo máximo en cinco o más campos temáticos (Ciencia de Materiales, Ciencias Agrícolas, Física, Geociencia, Ingeniería, Matemáticas y Química). Las publicaciones se concentran en los campos de la Física (267), Ingeniería (147) y Química (218), según datos de la Web of Science que comprenden el período de 1981-2003.

El universo de instituciones y académicos altamente especializados en el campo de la biotecnología es un valioso atributo para potenciar el desarrollo de dicha actividad, cuyo potencial justifica estudiar la interacción en red de estudiantes, empresarios, académicos e investigadores, su entorno, y las posibles implicaciones en el desarrollo de ese sector emergente.

Dichas interacciones son una caja negra: se observa un sistema que involucra un conjunto de datos de entrada prometedores, y otro conjunto de salida más o menos desalentador (escasas empresas o startups, patentes, modelos de utilidad y proyectos, pero muchos artículos de investigación), aunque virtualmente desconocemos las relaciones y procesos intermedios.

\footnotetext{
${ }^{3}$ Se conoce como Revolución verde al importante incremento de la productividad agrícola y por tanto de alimentos entre 1960 y 1980 en Estados Unidos, y extendida después por numerosos países. Consistió en la adopción de una serie de prácticas y tecnologías cuyo desarrollo inició en Sonora, México, dirigido por Norman E. Borlaug en 1943.
} 
La biotecnología reviste una importancia vital para Sonora, no sólo por tratarse de una zona semidesértica con grandes retos en materia de suelos, riego y condiciones climatológicas extremas, sino también porque estas condiciones pueden constituir áreas de oportunidad a partir de la biotecnología, misma en la que se adscriben gran parte de la investigación de frontera en la actualidad (Kafarski, 2012; Matyushenko, Sviatukha y Grigorova-Berenda, 2016).

El propósito de este trabajo es explorar la dinámica de las interacciones registradas entre la comunidad biotecnológica del estado de Sonora, para lo cual se aplica parcialmente un modelo basado en agentes inspirado en el SNA (Social Network Analysis) y en el SAC (Sistemas Adaptativos Complejos), propuesto por Beckenbach, Briegel y Daskalakis (2009) para identificar redes de innovación y signos de emergencia de los agentes económicos regionales. Se busca comprobar la conveniencia de esas herramientas para el estudio del comportamiento de variables clave al interior de un SRI (Sistema Regional de Innovación) en proceso de formación.

Los sistemas adaptativos complejos (SAC) constituyen un campo interdisciplinario de investigación que busca explicar cómo un gran número de entidades simples se organizan entre ellas, sin la ayuda de un control central, conformando un todo colectivo que crea patrones, usa información, y en algunos casos, evoluciona y aprende, y es cada vez más utilizado para comprender todo tipo de sistemas. El estudio de los SAC se centra en las propiedades complejas, emergentes y macroscópicas del sistema. Por su parte, la teoría de SNA (Social Network Analysis) nos proporciona las herramientas necesarias para 1) generar redes, 2) obtener métricas y 3) detectar clústeres.

Dichas herramientas facilitan la exploración de la caja negra antes referida, así como posibilitan poner a prueba nuestra hipótesis, en la que consideramos que los agentes que conforman el sector biotecnológico de la región operan de manera desarticulada, con un bajo índice de colaboración científica y escasa orientación hacia prácticas innovadoras, lo que afecta negativamente la eficiencia del SRI.

La naturaleza fundamental de la replicación de la evidencia empírica para la construcción del conocimiento científico motiva también la realización de este trabajo, al igual que la necesidad de reconstruir al menos parcialmente los procedimientos algorítmicos del estudio referido, mismos que no están disponibles públicamente.

El artículo se organiza en cuatro apartados, cuya primera parte examina los conceptos teóricos fundamentales de emergencia y complejidad dentro de los SRI, así como la importancia del enfoque del análisis de redes y los modelos basados en agentes. Seguidamente se discute la generación de redes dentro del sector biotecnológico regional, a partir de las cuales se obtiene un conjunto de datos que se traslada a un modelo basado en agentes. Este modelo se finca en la caracterización y segmentación de los agentes de acuerdo a una serie de categorías cuyo origen es la Teoría del Comportamiento Planeado de AjzenCarnegie. 
4 Emergencia y complejidad en los Sistemas Regionales de Innovación...

Arvizu Arvizu, M. A. y Bracamonte Sierra, Á.

En el cuarto apartado se aborda la aplicación del modelo y los resultados obtenidos, así como sus implicaciones para el sector biotecnológico del SRI de Sonora en particular, en un escenario caracterizado por una acelerada conversación de la cual es imperativo formar parte. Finalmente, se plantean conclusiones generales, así como recomendaciones para el enriquecimiento y profundización de la investigación basada en este tipo de modelos.

\section{EMERGENCIA Y COMPLEJIDAD EN LOS SISTEMAS REGIONALES DE INNOVACIÓN}

Los SRI funcionan como una compleja red de interacciones entre nodos que operan como agentes de intercambio de conocimiento y bienes de distinto orden, insertos dentro de una serie de sistemas (cultural, económico, etcétera) que funcionan bajo su propia lógica, es decir, que responden como un SAC. Este puede recibir distintos grados de incentivos que desencadenan diferentes e impredecibles respuestas. La Teoría de la Complejidad indica que un alto grado de estimulación produce una franja de actividad cercana al caos, siendo esa franja donde ocurre el fenómeno de emergencia. Excesiva estimulación produce caos, mientras que muy poca genera equilibrio o inercia.

En los SAC todo está en permanente cambio; dentro de ellos, los patrones siempre son dependientes del contexto (Taylor, 2003). La principal diferencia entre un Sistema Adaptativo Complejo y uno que no lo es, es la no linealidad derivada de la simple suma de sus partes, como ocurre, por ejemplo, en el caso del sistema financiero global (crisis, recesiones), o en el sistema inmunológico (fiebre, anticuerpos).

\section{Sistemas Regionales de Innovación desde la Teoría de Redes}

Los SRI pueden definirse como "lugares en los que las comunicaciones cercanas interfirma, las estructuras sociales y el ambiente institucional podrían estimular social y territorialmente el aprendizaje colectivo y la innovación continua" (Asheim y Isaksen, 2002, p. 85). Derivado de la Teoría General de Sistemas, los sistemas-red, y los estudios sobre distritos industriales e innovación (Cooke, 2008), el concepto es uno de los más aceptados en los círculos académicos y de políticas públicas (Carrillo y Contreras, 2015).

Estos sistemas juegan un rol central en la facilitación de los procesos de innovación (Molle y Djarova, 2009). Los sistemas están constituidos por redes, mismas que crean una proximidad intermedia cuyo espacio genérico es la región (Beckenbach et al., 2009).

Lo que distingue el actual momento de complejidad es la emergencia de una cultura de red (network culture) verdaderamente global (Taylor, 2003). Los SRI están sujetos a una serie de estímulos culturales y económicos (locales y globales) que hacen que la información viaje en tiempo real (la colaboración es o puede ser inmediata). El escenario es la región, pero "detrás de cámaras" las líneas de interacción trascienden latitudes y husos horarios. 
La importancia del estudio de los SRI desde la perspectiva de redes es continuamente mencionada en la literatura científica, pero ha sido poco abordada. Los trabajos suelen limitarse a asentar que es necesario el trabajo en red y la colaboración entre actores. Sin embargo, esta perspectiva proporciona herramientas para, más allá del inventario de infraestructuras y capacidades, colocar bajo el microscopio el universo de relaciones e intercambio de bienes tangibles e intangibles entre los agentes que conforman un sistema. Dicho enfoque permite espiar por el ojo de la cerradura, en lugar de sólo conformarnos con los componentes de entrada y de salida (Beckenbach et al., 2009), abandonando la mirada lineal.

\section{Conceptos básicos de emergencia y complejidad}

No existe una definición formal para describir un sistema complejo, aunque varias coinciden en los mismos elementos centrales. Para Holland (2006, p.1), los SAC son "sistemas compuestos por un amplio número de componentes, a menudo llamados agentes, que interactúan y se adaptan o aprenden”. Algunos ejemplos contemporáneos de problemas centrados en sistemas complejos son el cómo incentivar la innovación dentro de las economías dinámicas, la predicción de cambios en el sistema de transacciones global, la comprensión de los mercados, la preservación de ecosistemas, el control de Internet y el fortalecimiento del sistema inmunológico.

Melanie Mitchell, aunque advierte que se trata de una definición informal y de cierta manera circular, lo caracteriza como un sistema con un gran número de componentes que interactúan entre sí, en el que los componentes son relativamente simples en comparación con el sistema como un todo, y el cual no hay control central o comunicación global entre ellos y en el que la interacción da pie a un comportamiento complejo (Snodgrass, 2011). Según Holland (2006), los SAC comparten cuatro características fundamentales: 1) Paralelismo, dado que consisten en un gran número de agentes que interactúan enviando y recibiendo señales; 2) Acciones condicionales, pues las acciones de los agentes en un SAC usualmente dependen de las señales que reciben, esto es, los agentes tienen una estructura IF/THEN, por lo que si el vector de señal " $x$ " está presente, se ejecuta la acción " $y$ ", que puede ser también una señal, desatando complicados ciclos de retroalimentación, o una acción manifiesta dentro del ambiente del agente; 3) Modularidad, pues en un agente, un grupo de reglas a menudo se combina para formar subrutinas y 4) Adaptación y evolución, que implica que los agentes pueden cambiar con el tiempo y usualmente se trata de adaptaciones para mejorar el desempeño, no de cambios aleatorios.

El mejoramiento de "adaptación y evolución", la cuarta de estas características fundamentales, es actualmente objeto de investigación en NetLogo, un lenguaje utilizado para la simulación de SAC, para lo que se ha propuesto el método Agent Centric Montecarlo Cognition (ACMCC), el cual se centra en utilizar un modelo separado para la cognición de los agentes, con la ayuda de la extensión LevelSpace. Este modelo es utilizado 
6 Emergencia y complejidad en los Sistemas Regionales de Innovación...

Arvizu Arvizu, M. A. y Bracamonte Sierra, Á.

posteriormente por los agentes en el modelo primario para predecir los resultados de sus acciones, y así guiar su comportamiento.

\section{Emergencia dentro de los $S A C$}

La emergencia ocurre lejos del equilibrio y cerca del caos. En un SAC, un comportamiento emergente surge como respuesta a estímulos que pueden ser tanto intrínsecos como extrínsecos. Es el salto o gap, la región media que perturba lo que anteriormente se consideraba inmóvil, fragmenta lo que se consideraba verificado y muestra la heterogeneidad de lo que se imaginaba consistente consigo mismo (Taylor, 2003).

El comportamiento emergente, como el de un grupo de hormigas formando un puente interconectando sus propios cuerpos para salvar un obstáculo, es imposible de predecir, y no puede explicarse únicamente a través del estudio puntual de los componentes del sistema.

Las interconexiones entre los componentes de una red contienen la respuesta, pero esta no surge de manera aislada, sino que lo hace dentro de una alquimia combinatoria que constituye el objeto de investigación de frontera en el campo de la complejidad. Entramos en el terreno de lo impredecible. Dentro del universo observable, la única posibilidad es cuantificar la realidad evitando en la medida de lo posible el reduccionismo, y modelar patrones de comportamiento que si se pueden reconocer, llevan a identificar triggers o catalizadores, quizá no de un comportamiento emergente, pero sí de elección de modos de acción.

El método anteriormente descrito es el enfoque que se aplica en este trabajo como una aproximación al fenómeno de emergencia a través de la aplicación parcial de un modelo multiagente utilizando los datos de una pequeña muestra de nodos de la red de innovación del sector biotecnológico regional. El modelo fue desarrollado originalmente por Frank Beckenbach, Ramón Briegel y María Daskalakis en la Universidad Kassel, y es uno de los primeros intentos por incorporar conceptos de complejidad en el estudio de los Sistemas Regionales de Innovación y sus redes.

\section{REDES DE INNOVACIÓN ER Y SCALE FREE EN EL SECTOR BIOTECNOLÓGICO DE SONORA}

La diferencia entre los tipos de redes ER (Erdös y Renyi, o ER Networks) y las redes de escala libre (Scale Free Networks), está determinada por su topología. Mientras que las redes ER se trazan a partir de una variable asociacional aleatoria -o varias-, las redes de escala libre existen por sí mismas (Hein, Schwind y König, 2006). Un ejemplo de esto es la red de hipervínculos que conforman Internet, o una red de menciones dentro de un sistema de microblogging.

Dentro de las redes ER, los coeficientes de clusterización forman una curva normal, es decir, hay poca desviación, o todos los nodos están más o menos conectados dentro de los estándares, lo que no ocurre en las redes de escala libre, donde unos pocos nodos tienden a 
estar muy conectados, lo que genera un pico y una "cola gorda" (fat tail) de muchos nodos poco conectados. La diferencia está determinada por la manera en que se construyen ambos tipos de redes. Mientras que en una red ER la adhesión de nuevos nodos es más o menos uniforme entre los nodos ya existentes, en las redes de escala libre los nuevos nodos suelen adherirse con mayor frecuencia a los nodos que ya están altamente conectados (Hein et al., 2006).

Otra discrepancia es la influencia que una u otra topología tiene en el fenómeno de diseminación de la información. Mientras que en el modelo ER existen umbrales estadísticos bajo los cuales se detiene o no alcanza a afectar a todos los nodos, el resultado en las redes de escala libre es totalmente distinto. En este caso, técnicamente no existe un umbral, por lo que todos los nodos pueden ser afectados: trágico si es una epidemia, pero benéfico para la derrama de conocimiento.

Las redes de escala libre son una representación más cercana de las redes tal como se manifiestan "en el mundo real" que las tradicionales redes ER; sin embargo, son más difíciles de sujetar a modelos de simulación, especialmente si los nodos son personas.

\section{Aspectos metodológicos de la generación de las redes ER}

Sonora registra un alto grado de conectividad, pero la utilización de plataformas digitales sociales se reduce mayormente al intercambio y diseminación de información noticiosa, personal, cultural o de entretenimiento.

Son escasos los investigadores, profesores, estudiantes y/o profesionales que manejan una cuenta de Twitter para compartir ideas, conocimiento, eventos o datos de su especialidad, especialmente en STEM (Science, Technology, Engineering and Mathematics). Algunas instituciones de educación superior (IES) han adoptado este espacio vía sus departamentos de prensa, enfocándose en promover logros institucionales, y no exactamente en diseminar conocimiento. Esto dificulta que las distintas facultades y departamentos, a través de los mismos estudiantes y maestros, puedan tomar el control, compartir contenido útil y generar una conversación. ${ }^{4}$

En un principio, el diseño de la red ER se trazó en cuatro fases: 1) la creación de una red primaria conformada por las conexiones de investigadores miembros de cuerpos académicos tomados de las bases de datos de Promep en líneas de investigación afines a la biotecnología dentro de IES locales; 2) la ampliación de la red primaria mediante una lista de empresas

${ }^{4}$ La metodología original de este trabajo incluía la creación de una red de escala libre con ayuda de NodeXL; sin embargo, tuvo que ser descartada, pues 1) la tasa de respuesta de los cuestionarios enviados fue extremadamente baja y 2) la lógica del modelo de Beckenbach exigía un tipo de agente uniforme, en este caso, la firma. La ampliación del modelo para incluir distintos tipos de agente rebasa el alcance de esta investigación. No obstante, se espera desarrollarlo en un futuro trabajo de investigación. 
8 Emergencia y complejidad en los Sistemas Regionales de Innovación...

Arvizu Arvizu, M. A. y Bracamonte Sierra, Á.

generada a partir del Reniecyt, ${ }^{5}$ 3) la aplicación del método de bola de nieve a través de un cuestionario diseñado para los nodos de la red primaria, y 4) la incorporación de los nodos entre los que se identifiquen conexiones de la red de escala libre, ${ }^{6}$ también a través de la aplicación del cuestionario vía electrónica.

La metodología descrita pretendía descubrir una red híbrida que procuraba cubrir la academia o IES, actores relevantes del sector gobierno y empresa e incluso, incluir a la población estudiantil. Aunque la búsqueda de nodos se centraba en agentes potencialmente innovadores (investigadores SNI, empresarios de startups, generadores de patentes), no excluía a otros posibles agentes. La generación y el análisis de la red ER se realizó con apoyo del software UCINET, en el que se generó una matriz multiplex (tridimensional o multirrelacional) ligada a vectores de atributos.

\section{Análisis de la red ER}

Para la prueba piloto de la red ER se seleccionaron seis grupos de investigación consolidados y tres en vías de consolidación de las instituciones con mayor registro de actividad: Unison, CIAD e Itson. Nuestra variable aleatoria relacional consistía en el establecimiento de una conexión o relación del trabajo académico a través de grupos de investigación consolidados o en consolidación.

5 El Registro Nacional de Empresas Científicas y Tecnológicas (Reniecyt) contiene la información de los sujetos de los distintos fondos de apoyo del Consejo Nacional de Ciencia y Tecnología (Conacyt). Por su parte, el Programa de Mejoramiento del Profesorado (Promep) promueve la habilitación de docentes para lograr el perfil deseado en las instituciones de educación superior, es decir, docentes que realicen funciones de docencia, tutoría, generación de conocimiento y gestión.

${ }^{6} \mathrm{La}$ adopción de social media entre agentes innovadores en la región es muy limitada, por lo menos respecto a lo que sucede en otras latitudes. Lejos de constituir un nodo autónomo especializado de manejo de contenidos e interacciones, la "discusión" es un monólogo, el de la universidad. Como resultado, obtenemos una red de escala libre no sólo fragmentada, sino raquítica. Por este motivo, al menos regionalmente, las redes de escala libre sólo permiten complementar la información recopilada a través de la generación de una red tradicional, vía ejercicios que, empero, permiten el monitoreo de la evolución paulatina de la red, con el fin de enriquecer la comprensión de la misma. 
Cuadro 1. Actores del sector académico para la red ER de Biotecnología

\begin{tabular}{|c|c|c|c|c|}
\hline $\begin{array}{c}\text { Grupo de } \\
\text { investigación }\end{array}$ & $\begin{array}{c}\text { Nivel de } \\
\text { consolidación }\end{array}$ & Institución & $\begin{array}{l}\text { Biotech } \\
\text { code }\end{array}$ & $\begin{array}{l}\text { Lineas de } \\
\text { investigación }\end{array}$ \\
\hline $\begin{array}{l}\text { Biofísica y materia } \\
\text { condensada blanda } \\
\text { (Unison-CA-119) }\end{array}$ & Consolidado & $\begin{array}{l}\text { Universidad } \\
\text { de Sonora }\end{array}$ & GREY & $\begin{array}{l}\text { Biofísica y Físico } \\
\text { Química de } \\
\text { Materiales } \\
\text { Autoensamblantes } \\
\text { Física de fluidos } \\
\text { complejos } \\
\text { Química Física de } \\
\text { nanomateriales }\end{array}$ \\
\hline $\begin{array}{l}\text { Mecanismos de } \\
\text { defensa de las plantas, } \\
\text { transformación y } \\
\text { maduración de frutos }\end{array}$ & En consolidación & CIAD & GREEN & \\
\hline $\begin{array}{l}\text { Biotecnología y } \\
\text { productos naturales }\end{array}$ & Consolidado & Itson & GREEN & $\begin{array}{l}\text { Caracterización } \\
\text { bioquímica y } \\
\text { procesamiento de } \\
\text { productos }\end{array}$ \\
\hline $\begin{array}{l}\text { Ciencias de } \\
\text { Materiales. CA } 86\end{array}$ & Consolidado & $\begin{array}{l}\text { Universidad } \\
\text { de Sonora }\end{array}$ & $\begin{array}{l}\text { GREEN } \\
\text { (Nuevos } \\
\text { materiales) }\end{array}$ & $\begin{array}{l}\text { Química de polímero: } \\
\text { Polímeros } \\
\text { electroconductores } \\
\text { Biopolímeros } \\
\text { Materiales } \\
\text { compuestos con } \\
\text { matriz polimérica }\end{array}$ \\
\hline $\begin{array}{l}\text { Ciencias del Agua } \\
\text { (Itson-CA-03) }\end{array}$ & Consolidado & Itson & BLUE & $\begin{array}{l}\text { Hidroecología- } \\
\text { Ecohidrología }\end{array}$ \\
\hline $\begin{array}{l}\text { Biosistemas acuáticos } \\
\text { (itson-CA-42) }\end{array}$ & En consolidación & Itson & BLUE & $\begin{array}{l}\text { Biotecnología } \\
\text { Acuícola }\end{array}$ \\
\hline Biopolímeros & ND & CIAD & GREEN & ND \\
\hline $\begin{array}{l}\text { Desarrollo y Manejo } \\
\text { Sustentable de } \\
\text { Producción Acuícola }\end{array}$ & ND & CIAD & BLUE & ND \\
\hline $\begin{array}{l}\text { Biología y } \\
\text { sustentabilidad de } \\
\text { zonas áridas }\end{array}$ & ND & Unison & BROWN & ND \\
\hline
\end{tabular}

Fuente: Elaboración propia con información de la base de datos de cuerpos académicos reconocidos por Promep y trabajo de campo de revisión de la información proporcionada por Unison, CIAD e Itson en sus respectivas páginas de Internet. 
Dada la escasa participación de los agentes involucrados, se descartó la posibilidad de generar una red de agentes a nivel individuo emanados de todos los componentes de la triple hélice, y se diseñó un segundo cuestionario enfocado solamente a la exploración de nodos a nivel representativo (firma), en el cual se recoge información sobre diversos atributos de la misma (percepciones anuales, intención de ampliación de cuota de mercado, confianza en socios regionales) y los individuos que la conforman (curiosidad, especialización del conocimiento). Esta decisión facilitó la aplicación de la lógica centrada en la firma del modelo de Beckenbach et al. (2009), pese a limitar el número de agentes disponibles regionalmente. Los agentes identificados se presentan en el cuadro 2.

Uno de los temas que se tocan en el proyecto de investigación del que se deriva este trabajo es que la falta de disposición hacia la colaboración y participación interdisciplinaria entre los agentes del SRI en estado de formación afecta negativamente el desempeño de éste, situación que es necesario cambiar.

Cuadro 2. Actores regionales y capacidades locales dentro del ramo biotecnológico

\begin{tabular}{|c|c|c|c|c|}
\hline & IES & Gobierno & Empresa & $\mathrm{AC}$ \\
\hline $\begin{array}{l}\text { Biotecnología } \\
\text { Café }\end{array}$ & $\begin{array}{l}\text { Unison, Itson, } \\
\text { IGI Estación } \\
\text { Regional }\end{array}$ & $\begin{array}{l}\text { Centro de } \\
\text { Investigaciones } \\
\text { Agrícolas del }\end{array}$ & $\begin{array}{l}\text { Hermosillo } \\
\text { Drone Lab }\end{array}$ & \\
\hline $\begin{array}{l}\text { Biotecnología } \\
\text { desértica } \\
\text { Espacio y } \\
\text { geomicrobiología } \\
\text { Biotecnología de } \\
\text { zonas áridas y } \\
\text { desérticas }\end{array}$ & Noroeste & $\begin{array}{l}\text { Noroeste (CIANO), } \\
\text { Secretaría de } \\
\text { Agricultura y } \\
\text { Desarrollo Rural } \\
\text { (Sagarpa) }\end{array}$ & & \\
\hline $\begin{array}{l}\text { Biotecnología } \\
\text { Verde/Gris } \\
\text { Agricultura } \\
\text { Medio Ambiente }\end{array}$ & Unison, Itson & $\begin{array}{l}\text { Laboratorio } \\
\text { Nacional de } \\
\text { Sistemas de } \\
\text { Concentración y } \\
\text { Química Solar, } \\
\text { Comisión de } \\
\text { Energía del Estado } \\
\text { de Sonora } \\
\text { (COEES), Secretaría } \\
\text { de Educación } \\
\text { Pública (SEP/SES), } \\
\text { Fundación Produce }\end{array}$ & $\begin{array}{l}\text { Sunbionics, } \\
\text { Solarscape de } \\
\text { México S.A. de } \\
\text { C.V., Maeve } \\
\text { Tecnología } \\
\text { productores } \\
\text { especializados de } \\
\text { energía S.A. de } \\
\text { C.V., Globalmet } \\
\text { Sapi de C.V., } \\
\text { Citro-Des }\end{array}$ & $\begin{array}{l}\text { Patronato para la } \\
\text { Investigación y } \\
\text { Experimentación } \\
\text { Agrícola del } \\
\text { estado de Sonora, } \\
\text { AC (PIEAES), } \\
\text { Citro-Des }\end{array}$ \\
\hline
\end{tabular}




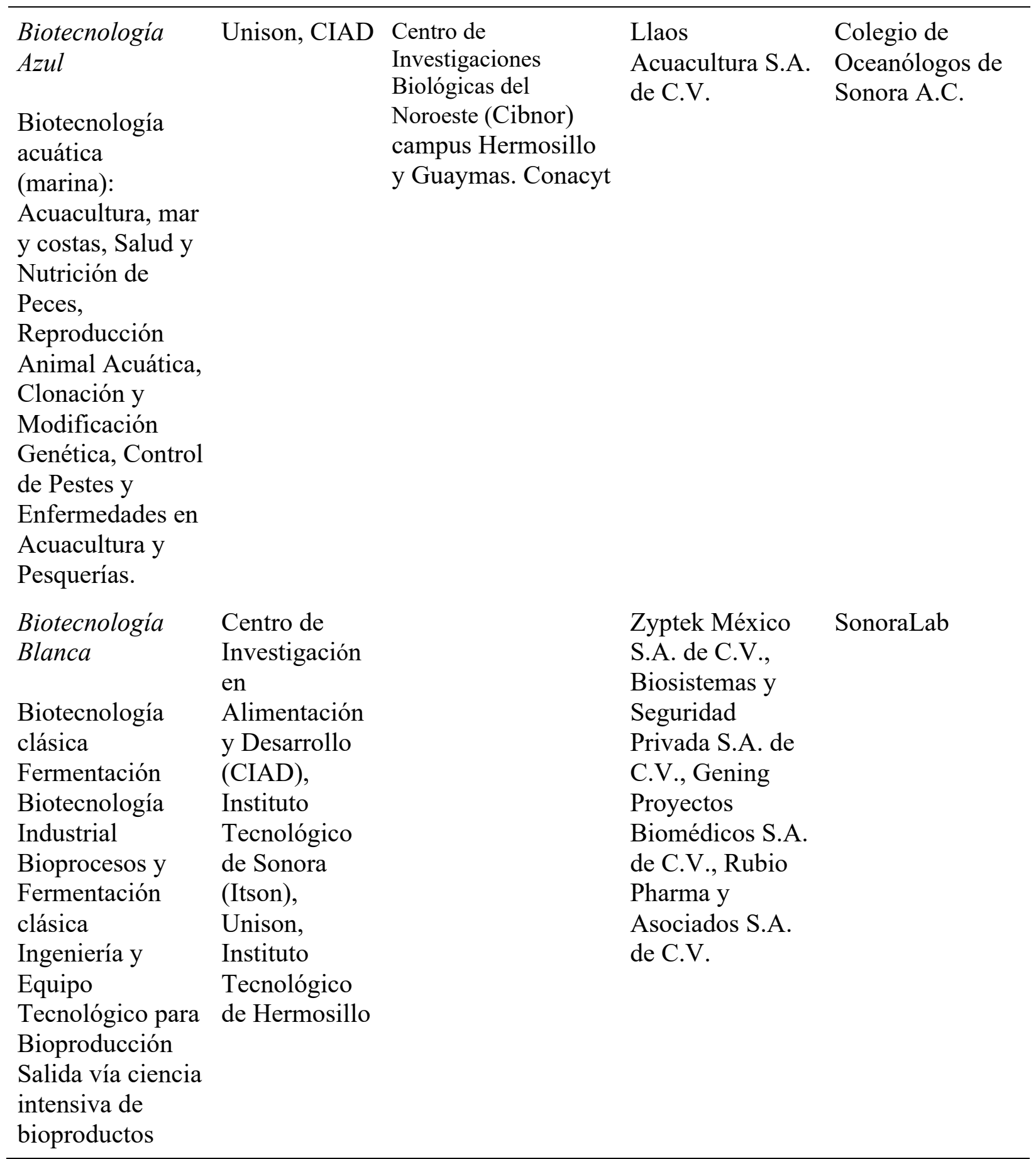

Fuente: Elaboración propia con base en la clasificación del código arcoíris de la biotecnología, consulta de las bases de datos del Registro Nacional de Instituciones y Empresas Científicas y Tecnológicas (Reniecyt) y trabajo de campo.

A partir de la información recopilada se obtuvo la red desplegada en la figura 1, donde según los datos de medidas de centralidad especificados en el cuadro 3, firmas como Rubio Pharma, Agropro y Galaz Science y Engineering se encuentran en posiciones privilegiadas 
12 Emergencia y complejidad en los Sistemas Regionales de Innovación...

Arvizu Arvizu, M. A. y Bracamonte Sierra, Á.

en términos de centralidad intermedia, seguidas de Livestock Analytics y Sunbionics. En el lado opuesto, aparentemente aisladas, aparecen las firmas Gening, Globalmet y PROAX.

El grado de centralidad (degree) mide el número de lazos con los que un agente está conectado a otros. El grado de centralidad mínimo es 0 , si un agente no tiene conexiones; el máximo está dado por $\mathrm{d}=\mathrm{g}-1$ ( $\mathrm{g}$ siendo el número de nodos). La centralidad intermedia de una red está dada por la tasa del número actual de lazos entre los agentes y el número total de $\operatorname{nodos}(\mathrm{g})$.

La mayoría de las firmas seleccionadas para la aplicación de la encuesta son consideradas innovadoras en virtud de que han sido acreedoras de apoyos por parte del PEI (Programa de Estímulo a la Innovación) otorgados por el Conacyt en una o varias ocasiones. En otros casos, se consideran innovadoras debido al área emergente a la que se dedican.

Figura 1. Red de agentes del sector biotecnológico
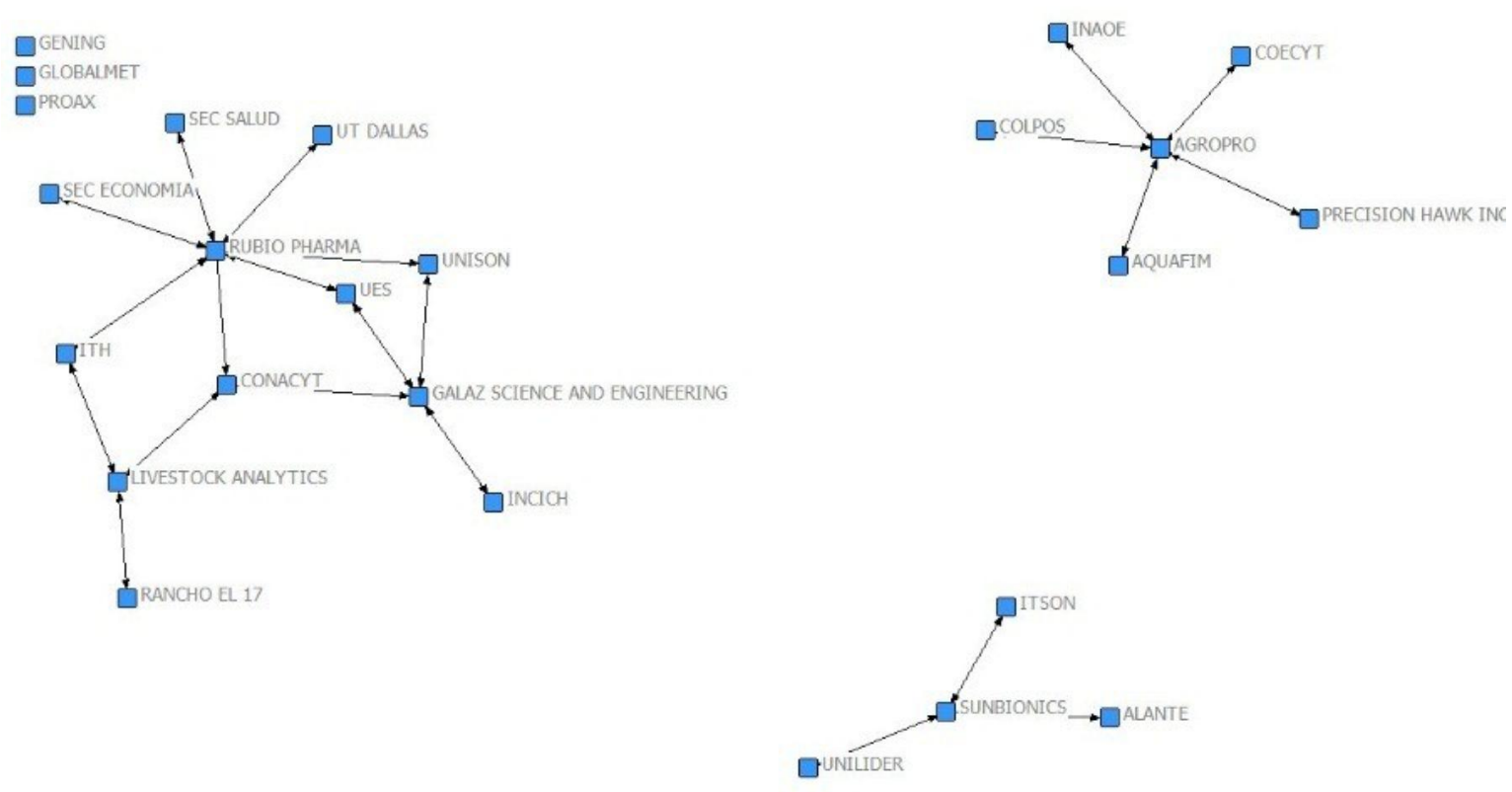

Fuente: Elaboración propia/Ucinet 6 
Cuadro 3. Medidas de centralidad para la red de actores del ramo biotecnológico

\begin{tabular}{|c|c|c|c|c|c|c|}
\hline & Degree & Betweenness & Closeness & $\begin{array}{l}\text { Harmonic } \\
\text { Closeness }\end{array}$ & Eigenvector & $\begin{array}{l}\text { 2-Local } \\
\text { Eigenvector }\end{array}$ \\
\hline Gening & 0 & 0 & 625 & 0 & 0 & 0 \\
\hline Globalmet & 0 & 0 & 625 & 0 & 0 & 0 \\
\hline PROAX & 0 & 0 & 625 & 0 & 0 & 0 \\
\hline Alante & 1 & 0 & 555 & 2 & 0 & 3 \\
\hline Unilider & 1 & 0 & 555 & 2 & 0 & 3 \\
\hline Itson & 1 & 0 & 555 & 2 & 0 & 3 \\
\hline $\begin{array}{l}\text { Precision Hawk } \\
\text { Inc. }\end{array}$ & 1 & 0 & 509 & 3 & 0 & 5 \\
\hline Aquafim & 1 & 0 & 509 & 3 & 0 & 5 \\
\hline Coecyt & 1 & 0 & 509 & 3 & 0 & 5 \\
\hline Colpos & 1 & 0 & 509 & 3 & 0 & 5 \\
\hline INAOE & 1 & 0 & 509 & 3 & 0 & 5 \\
\hline Rancho el 17 & 1 & 0 & 385 & 4.17 & 0.09 & 3 \\
\hline INCICH & 1 & 0 & 383 & 4.42 & 0.14 & 4 \\
\hline Sec. Economía & 1 & 0 & 377 & 5.17 & 0.23 & 7 \\
\hline Sec. Salud & 1 & 0 & 377 & 5.17 & 0.23 & 7 \\
\hline UT Dallas & 1 & 0 & 377 & 5.17 & 0.23 & 7 \\
\hline ITH & 2 & 5.33 & 373 & 6.08 & 0.31 & 10 \\
\hline UES & 2 & 3.17 & 373 & 6.08 & 0.36 & 11 \\
\hline Unison & 2 & 3.17 & 373 & 6.08 & 0.36 & 11 \\
\hline Sunbionics & 3 & 3 & 553 & 3 & 0 & 3 \\
\hline Livestock Analytics & 3 & 11 & 375 & 6 & 0.16 & 6 \\
\hline Conacyt & 3 & 14.33 & 369 & 7 & 0.45 & 14 \\
\hline $\begin{array}{l}\text { Galaz Science And } \\
\text { Engineering }\end{array}$ & 4 & 12.83 & 373 & 6.67 & 0.25 & 8 \\
\hline Agropro & 5 & 10 & 505 & 5 & 0 & 5 \\
\hline Rubio Pharma & 7 & 35.17 & 367 & 8.67 & 0.41 & 12 \\
\hline
\end{tabular}

Fuente: Elaboración propia/Ucinet 6. 
14 Emergencia y complejidad en los Sistemas Regionales de Innovación...

Arvizu Arvizu, M. A. y Bracamonte Sierra, Á.

El agente gubernamental con un mayor índice de centralidad es, sin duda, Conacyt. En cuanto a IES, los que suman un mayor número de conexiones son el Instituto Tecnológico de Hermosillo (ITH), la Universidad Estatal de Sonora (UES) y la Universidad de Sonora (Unison). Las IES ITH y UES, a pesar de no ser consideradas inicialmente dentro de la exploración de agentes del sector biotecnológico, adquirieron prominencia durante la fase de aplicación del cuestionario, al ser mencionadas por las firmas encuestadas como agentes con los que establecen relaciones de cooperación.

Si bien este conjunto de datos es pequeño aún y no permite conclusiones definitivas, sí muestra indicios de la concentración de actividad en red en términos de innovación. El pequeño conjunto de agentes al que puede accederse actualmente de manera formal podrá ampliarse conforme aparezcan en la región un mayor número de startups y firmas innovadoras dedicadas al ramo de la biotecnología, lo que permitirá enriquecer la escasa información disponible, así como alimentar el modelo con nuevos datos.

Una ampliación de este modelo con un aparato logístico capaz de incluir agentes distintos a la firma, especialmente IES, sería muy útil en el estudio de SRI en proceso de conformación en regiones como el estado de Sonora, donde una buena parte de la actividad en el ramo biotecnológico está todavía concentrada allí.

Algunas de las firmas aquí mostradas establecen estrechos lazos de colaboración con universidades e instituciones de gobierno, los cuales se expresan en convenios institucionales, asesorías, intercambio de laboratorios, estancias y residencias, entre otros. En ocasiones el lazo es doble o triple, de manera que la firma no sólo tiene convenio institucional con una institución de educación superior, sino que además realiza intercambio de laboratorio, así como estancias y residencias, al tiempo que mantiene relaciones con otras firmas e instituciones. Otras firmas manifestaron no sostener ningún lazo.

El cuestionario propone las siguientes formas de colaboración con otras firmas, instituciones gubernamentales e IES: 1) Mentor, 2) Intercambio de textos científícos, 3) Intercambio de laboratorio, 4) Intercambio de materiales, 5) Asesoría, 6) Coautoría de textos, 7) Convenio institucional y 8) Estancias y residencias; algunas firmas, que aparecen sin lazos en la figura 1, dejaron en blanco este último apartado.

El Análisis de Redes Sociales no debería limitarse a ejercicios de evaluación descriptiva y exploratoria, puesto que juegan un rol muy importante dada la generación de indicadores cuantitativos de red de alto valor tanto a nivel firma y clúster, incluyendo los de centralidad en estimados econométricos que podrían ayudar a medir el impacto de un CDP (Cluster Development Program) en una red inter organizacional, la misma red considerada responsable de la efectividad de un programa de clústeres (Giuliani y Pietrobelli, 2011). La implementación de una evaluación de esta naturaleza requiere, empero, de un estudio longitudinal que sobrepasa los alcances de este trabajo, problema del que surge la necesidad de la construcción de modelos que ayuden a evaluar el comportamiento de las redes de las distintas entidades que componen un clúster en formación, o un SRI en su conjunto, de 
acuerdo con las variables y parámetros observables actualmente, y en el que se pueden introducir elementos con el fin de probar el impacto de éstos en el desempeño de los agentes en el mediano y largo plazo.

Dichos modelos permiten alejarnos de un pensamiento orientado al estéril recuento de los componentes de un clúster naciente o un SRI apenas en formación, concentrando esfuerzos en análisis prospectivos que permitan obtener información útil para la creación de políticas públicas optimizadas, con una mejor comprensión de las ventajas y limitaciones de las diferentes posiciones y estructura de la red.

El estudio completo del que se deriva este trabajo aborda además del sector biotecnológico los sectores TIC y aeroespacial en el estado de Sonora.

\section{APLICACIÓN DE UN MODELO BASADO EN AGENTES DENTRO DE UN SRI}

La red descrita anteriormente representa solamente un recorte en el tiempo, es decir, una especie de fotografía del espacio temporal en el que se realizó la investigación. Si bien guarda información sobre 1) la topología de la red formada por los agentes, 2) los atributos de estos a nivel firma y 3) los múltiples contenidos que demarcan cada lazo, no nos permite adentrarnos en su morfología, y por tanto, en las diferentes retroalimentaciones entre los estados de los agentes y la red como un todo (Beckenbach et al., 2009).

La única manera de penetrar este "nuevo mundo" en el que la red se comporta de manera dinámica es utilizando un modelo de simulación, en el que los atributos o la información empírica de los agentes son convertidos en variables, cuyo conjunto determina un estado de la firma. Así, los agentes son capaces de reaccionar ante un estímulo, retroalimentación o umbral determinado por las reglas del modelo, evolucionando a través de cada ciclo o iteración.

Para ilustrar el proceso de aplicación de un modelo basado en agentes, utilizamos el modelo propuesto por Beckenbach, et al. (2009), el cual fue aplicado a gran escala en Alemania. Creemos que constituye una aproximación útil en regiones emergentes, porque aunque se carece de la información necesaria para la aplicación de encuestas de innovación estandarizadas, permite profundizar en aspectos relevantes para los procesos de innovación -como la inclinación hacia la cooperación científica y la confianza en socios regionales-.

Beckenbach, Briegel y Daskalakis (2007) identifican cuatro aspectos del comportamiento relevantes para los procesos de innovación: 1) el conocimiento declarativo y procedural, 2) las habilidades (en términos de encontrar nuevas heurísticas y capacidades de recombinación y asociación de los elementos de conocimiento dados), 3) la motivación intrínseca y extrínseca y por último, 4) los rasgos de la personalidad (curiosidad, aceptación del riesgo, etcétera). 
La interacción entre las dimensiones social, competitiva e individual del proceso de innovación puede caracterizarse estableciendo una tipología de agentes y una tipología de competencia. Por ejemplo, un tipo de agente puede ser caracterizado con una combinación de expectativas pesimistas, objetivos de innovación del tipo reactivo, y cierta lealtad a los paradigmas; mientras que un tipo de competencia, por ejemplo, puede implicar un bajo grado de concentración, bajas barreras de entrada y la orientación hacia la dimensión del costo (Daskalakis, 2016).

\section{Trabajando en equipo con la psicología: La personalidad innovadora}

La innovación es un modo específico de acción. No ocurre "de la nada"; es decir, no se genera automáticamente por condiciones competitivas de mercado. Requiere agentes dispuestos a innovar y promover las actividades correspondientes, abandonando otros modelos de acción (Beckenbach et al., 2009).

Para los autores, distinguir a estos agentes con la etiqueta binaria de "innovadores" o "no innovadores" es demasiado simplista dentro del contexto de un SRI. El análisis cuidadoso de un sistema regional requiere tomar en cuenta a todos los agentes involucrados, sus diferentes modos de acción y su incrustamiento en los distintos mecanismos de coordinación.

Más aún, cada uno de los agentes tiene motivaciones, capacidades y limitaciones para actuar dentro de un contexto en red. Los múltiples modos de acción (rutina, búsqueda por imitación, búsqueda por innovación) compiten entre sí para ser activados por el agente debido a fuerzas generadas de manera endógena.

\section{Aproximación multimodo con base en las escuelas Ajzen y Carnegie}

En el contexto de los SRI existen dos requerimientos para la explicación conductista: 1) los diferentes modos de acción específicos de cada capa del SRI (rutina, imitación, innovación) deben ser parte del explanandum y 2) la explicación debería ser empíricamente significativa (Beckenbach et al., 2009).

Beckenbach et al. (2009) señalan que no existe un concepto en la literatura científica conductista que satisfaga ambos requerimientos, por lo que propone sintetizar dos aproximaciones bien conocidas, cada una de las cuales ha sido aplicada a problemas empíricos y ha sido utilizada para explicar más de un modo de acción. Estas aproximaciones son, por un lado, la escuela Ajzen, con la TPB (Theory of Planned Behavior, o Teoría del Comportamiento Planeado), y por otro, la Economía del Comportamiento (Behavioral Economics). 
Cuadro 4. Los enfoques Ajzen y Carnegie

$$
\text { Ajzen }
$$

Carnegie

El enfoque de Ajzen es explicar actividades Esta aproximación arroja luz sobre dos intencionales, es decir, actividades resultantes de modos de decisión usualmente pasados un plan consciente para hacer algo. por alto: rutinas y búsqueda.

Este plan o intención está influenciado por tres factores cognitivos:

1. La actitud del agente sobre los atributos de la actividad planeada.

2. Lo apropiado de esta actividad para las normas sociales perseguidas por el agente.

3. La habilidad del agente para manejar o controlar dicha actividad.

Fuente: Adaptado de Understanding Complex Systems (2009).

Figura 2. Diagrama de cadena causal para seleccionar un modo de acción

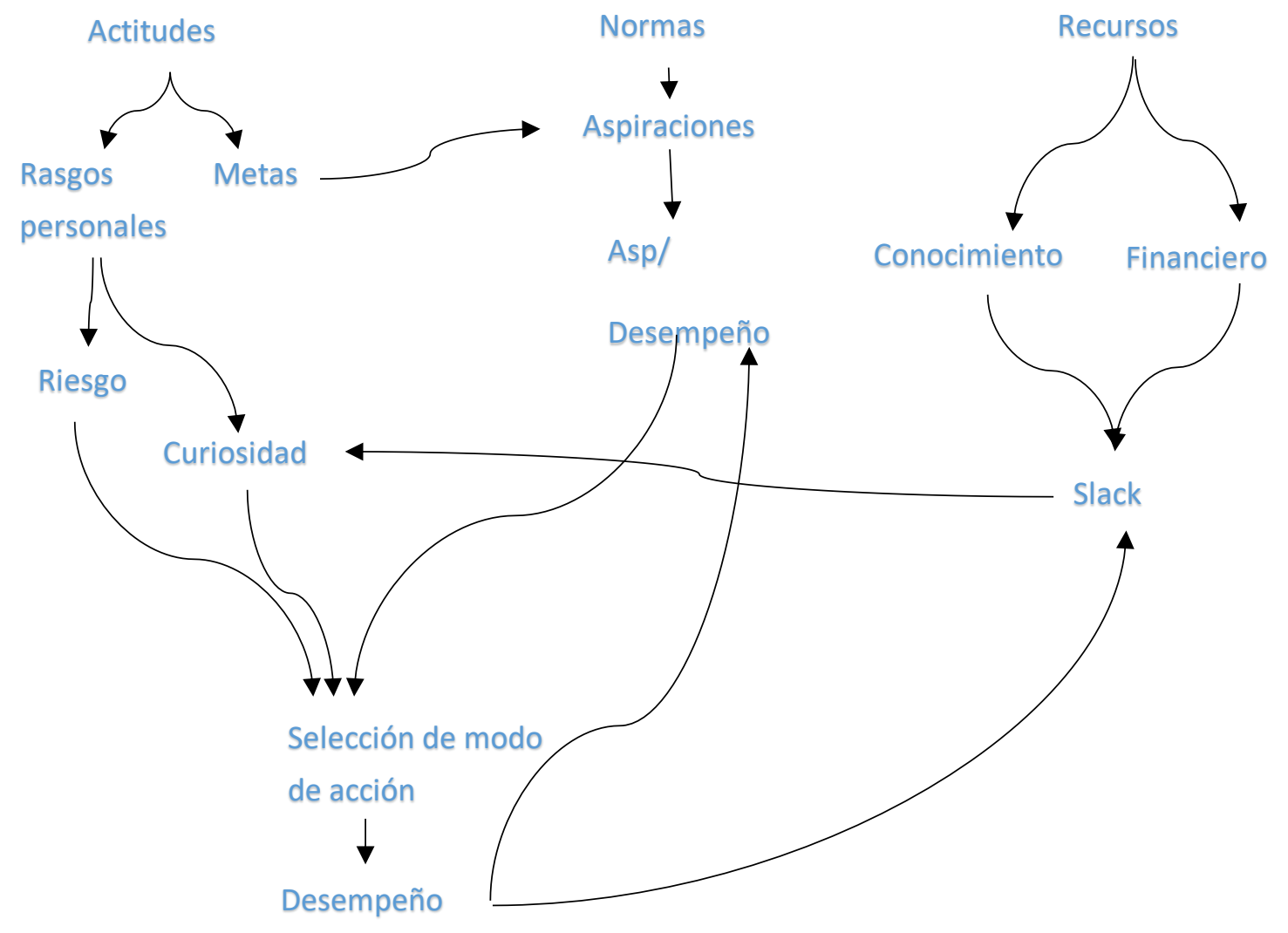

Fuente: Understanding Complex Systems (2009). 
De acuerdo con el diagrama presentado en la figura 2, la selección de un modo de acción está determinado por actitudes, reflejadas en inclinaciones de la personalidad (aversión al riesgo, curiosidad) y metas, así como por normas, las cuales están estrechamente relacionadas con la consecución de estas metas. Metas de alto perfil se traducen en aspiraciones que a su vez tendrán como resultado una determinada tasa de desempeño, constreñida por distintos factores. El desempeño resultante de la selección de modo de acción retroalimenta esta tasa a medida que avanzan las iteraciones, lo que eventualmente puede afectar tanto la selección del modo de acción como el desempeño en sí mismo. Por otra parte, los activos intelectuales y financieros generan un componente llamado "slack". Éste representa un margen de maniobra que puede ser alto o bajo y, aunado al componente de la curiosidad, afecta también el subsecuente modo de acción y a su vez, el futuro desempeño del agente.

\section{Modelo ajustado a las necesidades de un SRI en estado emergente}

Las limitaciones derivadas de un SRI incipiente, tales como la ausencia de métricas y la dificultad para registrar la totalidad de las variables propuestas por los manuales de Oslo y Bogotá, conducen a la búsqueda de una alternativa no para "medir" la innovación, sino para explorar el potencial innovador.

Si bien se renuncia temporalmente a la homogeneidad que permitiría la comparación de resultados a escala global, la utilización de un modelo ajustado a las características de la región proporciona información de mayor utilidad para aplicación de políticas públicas efectivas. Así, en lugar de utilizar la perspectiva usual top-down, la investigación se reorienta hacia una perspectiva bottom-up, preocupada por las motivaciones y capacidades del agente, así como sus limitaciones para actuar en un contexto de red.

En el modelo propuesto por Beckenbach et al. (2009), las subredes interconectadas que conforman la red son subcapas en las que se distingue una primera capa exterior, que consiste en las normas políticas e institucionales de la región compartidas por todos los agentes; una capa media, que aglomera agentes interesados en innovar y una núcleo, en la que se concentran los agentes innovadores (como si fuera una cebolla).

En este contexto, los modos de acción son: 1) rutina, 2) imitación y 3) innovación, donde este último puede ser individual o colaborativo. Los principales ajustes del modelo con respecto al utilizado por Beckenbach et al. (2009) conciernen al tipo de población y tamaño de muestra. El equipo de Beckenbach aplicó un cuestionario a más de mil compañías ubicadas en Kassel, es decir, se centra en la firma como agente. Nuestra pretensión inicial de incluir en este modelo a todos los actores que intervienen en un SRI debió abandonarse, por lo que gobierno y academia debieron excluirse. Por otra parte, el número de firmas encuestadas es significativamente menor, debido sencillamente a una todavía escasa población de firmas dedicadas al ramo dentro de la región.

Un segundo ajuste se realizó con respecto a la cantidad de componentes codificados en el modelo, puesto que una codificación completa no solo rebasa los alcances de este trabajo, 
sino que oscurece uno de sus objetivos principales, que es contribuir a la comprensión y réplica de la construcción de un modelo microeconómico basado en agentes dentro de un SRI utilizando el lenguaje de programación NetLogo.

La simulación del modelo multiagente se realiza utilizando NetLogo, software de código abierto y acceso libre, el cual se obtuvo durante el curso de "Introducción a la complejidad" llevado a cabo por el Instituto Santa Fe, bajo la dirección de la doctora Melanie Mitchell, a través del sistema de aprendizaje en línea Complexity Explorer.

\section{APLICACIÓN DEL MODELO Y RESULTADOS}

La implementación del modelo se realizó con base en un conjunto de ocho agentes utilizando variables que miden actitudes (aversión al riesgo, curiosidad, metas) y normas (utilidades aspiradas, utilidades obtenidas), las cuales motivan la elección de uno u otro modo de acción, así como variables de activos (endowmentt) intelectuales y económicos de la firma (conocimiento especializado, capacidad financiera). El criterio para la selección de un modo de acción inicial está modulado por tres parámetros del comportamiento (actitud aspiracional $\beta$, propensión a imitar y propensión a cooperar).

A continuación se describen las características del modelo:

Si denotamos

$\mathrm{g}$ - ingresos obtenidos el último ciclo o iteración

s- nivel de aspiración correspondiente

El modo de acción está determinado por los siguientes criterios:

$$
\begin{gathered}
\frac{g}{s} \geq \beta \text { : rutina } \\
\beta-i \leq \frac{g}{s}<\beta: \text { imitación } \\
\beta-x \leq \frac{g}{s}<\beta-i \text { : innovación cooperativa } \\
\frac{g}{s}<\beta-x: \text { innovación individual }
\end{gathered}
$$

Tenemos que la selección del modo de acción opera como una función de la tasa ingresos/nivel de aspiración. Por tanto, si la firma aspira a ganar 100 pesos durante el trimestre, y el último trimestre ganó 200, es decir el doble, la tasa es mayor a la actitud aspiracional, y por tanto se elige el modo de acción rutina, o mantener las cosas tal como están. Si por el contrario, dicha tasa es menor que la actitud aspiracional -es decir, la firma obtiene ingresos menores a los aspirados-, pero mayor que el resultado de restar el valor 
asignado a la variable $\mathrm{i}$ (propensión a imitar), ${ }^{7}$ a la actitud aspiracional, se decanta por el modo de acción imitación. Por ejemplo, si la firma deseaba ganar 100, pero sólo obtuvo 50, y la propensión a imitar es alta, existe motivación para incursionar con un nuevo producto, aunque sólo sea una copia de otros ítems en el mercado. En el caso de los modos de innovación cooperativa e individual, interviene además la variable x (propensión a cooperar).

Lo anterior se consigue con el siguiente código:

ask turtles [

if $g / s>=B$ [set color blue ] ; rutina

if $g / s<B$ and $g / s>=B-i$ [set color pink] ;; imitación, $i$ es la propensión a imitar

if $g / s<B-i$ and $g / s>=B-X$ [set color yellow]

;; innovación cooperativa, X es la propensión a la cooperación

if $g / s<B-X$ [set color red] ;; innovación individual

\section{forward 3}

En este punto, al ejecutar el código anterior obtenemos en pantalla el número total de agentes (turtles), cada uno identificado con un color que indica el modo de acción elegido de acuerdo con los parámetros obtenidos a través del cuestionario de investigación.

Contrario a la actitud aspiracional $\beta$, el nivel aspiracional se actualiza al final de cada paso según la ecuación:

$$
s(t+1)=(1-\Phi) s(t)+\Phi g(t)
$$

Donde $\Phi$ representa la flexibilidad de adaptación (de cada empresa). La expresión anterior se expresa dentro de NetLogo de la manera siguiente:

$$
\text { set } s 1((1-f a) * s)+(f a * g)
$$

set $s$ s

Así, utilizando estas expresiones y el conjunto de datos disponible, observamos que en el caso de las empresas estudiadas dentro del sector biotecnológico de Sonora, inicialmente la mayoría se ubica dentro del modo de acción de innovación cooperativa. Para la iteración 20 por ciento, 50 por ciento de las compañías se desplaza al modo rutina, mientras que 50 por

${ }^{7}$ Este valor, al igual que el de $\mathrm{x}$, varía para cada firma y se obtiene a partir de las respuestas obtenidas a las preguntas incluidas en el cuestionario de investigación. 
ciento restante permanece en modo de innovación cooperativa, y para la iteración 30, la totalidad de las compañías operan en modo rutina, comportamiento que se repite en adelante.

Las variables i y x, que reflejan respectivamente la propensión a imitar y la propensión a cooperar de cada empresa, están dadas por las respuestas a un conjunto de preguntas asociadas a dichos fenómenos dentro del cuestionario de investigación.

Figura 3. Resultados de elección de Modos de Acción

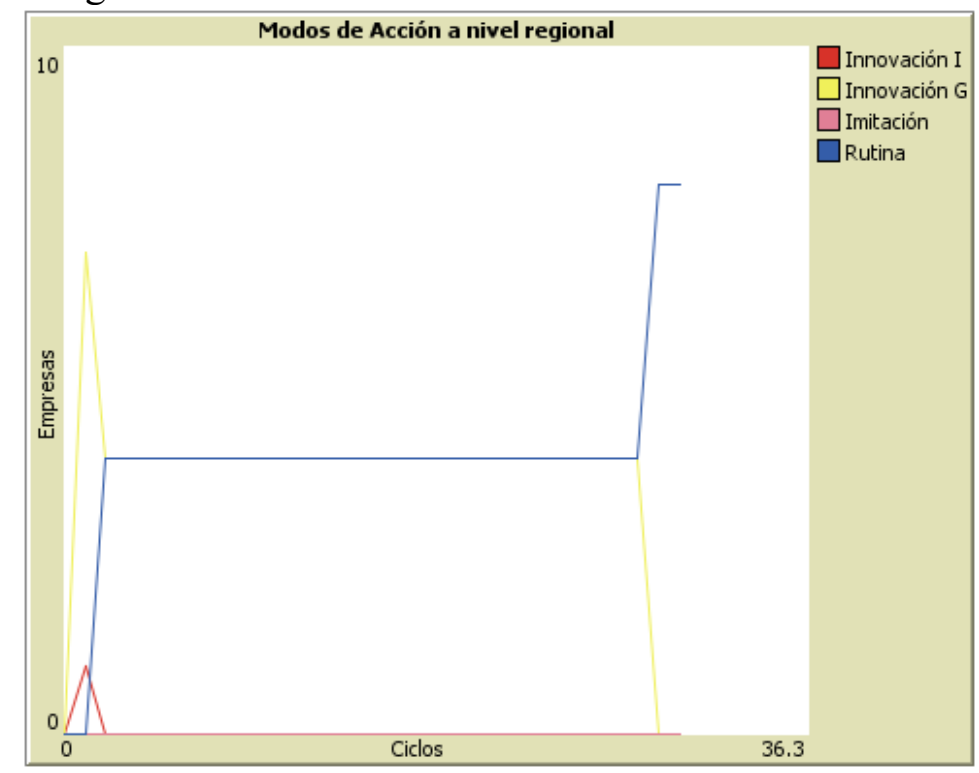

Fuente: Elaboración propia/NetLogo 5.3.1

Utilizando la notación de Beckenbach et al. (2009), el TC (Costo de Transacción) de una red de innovación es una función de la especificidad del conocimiento (s), el número de componentes del conocimiento (q) y la confianza (r) en términos de la frecuencia de transferencias exitosas de conocimiento en el pasado.

$$
T C=f(s, q, r)
$$

La F2 (Fuerza Innovadora), se caracteriza por:

$$
F 2=\frac{\alpha f 0+f 1+f 2}{\operatorname{cin}}
$$

Donde $\alpha$ representa la aceptación del riesgo, cin el costo esperado de la innovación, y f (i) la curiosidad o inclinación por la exploración con el valor 0 , la aspiración de ganancias con el valor 1, y la aspiración de cuota de mercado con el valor 2 .

Por otra parte, $F 1$ (Fuerza Imitadora) está determinada por:

$$
F 1=\frac{f 1+f 2}{\operatorname{cim}}
$$


22 Emergencia y complejidad en los Sistemas Regionales de Innovación...

Arvizu Arvizu, M. A. y Bracamonte Sierra, Á.

Y F0 (Fuerza de preservación o Rutina) es igual a 1.

Para obtener $F 2$, es necesario calcular

$$
\begin{aligned}
& f 0=w 0(k r+f r) \\
& f 1=w 1\left(\frac{a s p}{p}\right)^{E 1} \\
& f 2=w 2\left(\frac{a s m}{m}\right)^{E 2}
\end{aligned}
$$

Donde $w 0$ representa la inclinación por la exploración, $k r$ las reservas de conocimiento, $f r$ las reservas financieras, asp el nivel de ganancias aspiradas y asm el nivel de cuota de mercado aspirado. $E 1$ y $E 2$, parámetros utilizados para la elasticidad del mercado, se utilizan con los valores 8 y 16 en la constelación paramétrica estándar de referencia utilizada por

\begin{tabular}{|c|c|c|c|c|c|}
\hline \multirow[b]{2}{*}{ Variable } & \multicolumn{5}{|c|}{$\begin{array}{l}\text { Medias derivadas } \\
\text { empíricamente }\end{array}$} \\
\hline & Parámetro & $F \_I R$ & $F_{-} I I M$ & $F_{-} R O U T$ & Escala \\
\hline Aceptación del Riesgo & $\alpha$ & 4 & 5 & - & $1-5$ \\
\hline $\begin{array}{l}\text { Inclinación por la } \\
\text { Exploración }\end{array}$ & & 4.5 & 4 & - & $1-5$ \\
\hline Aspiración de utilidades & & 3.6 & 4 & - & $1-5$ \\
\hline $\begin{array}{l}\text { Aspiración de cuota de } \\
\text { mercado }\end{array}$ & & 4.6 & 5 & - & $1-5$ \\
\hline $\begin{array}{l}\text { Propensión a la } \\
\text { cooperación }\end{array}$ & $\mathrm{X}$ & 4.8 & 5 & - & $1-5$ \\
\hline Confianza regional & & 4.5 & 4.5 & - & $1-5$ \\
\hline
\end{tabular}
Beckenbach et al. (2009).

Tabla 1. Calibración estadística de los parámetros de comportamiento del modelo

Fuente: Elaboración propia con base en el conjunto de datos generado a partir del cuestionario de investigación enviado a las firmas del sector biotecnológico de Sonora.

Para derivar las medias relevantes es necesario distinguir diferentes tipos de comportamientos de firmas. En relación al modelo, se clasifican tres tipos: Innovadores radicales, imitadores y firmas que operan bajo rutinas. En el caso de Sonora, la distribución de los tipos de firmas se distribuye como sigue: 80 por ciento se ubica en la categoría $F_{-}$IR (innovadores), mientras que 20 por ciento lo hace dentro de la categoría de imitadores 
(F_IIM). Ninguno de los encuestados se identificó como firma que no conduce ningún tipo de innovación (F_ROUT).

Al tratarse de un sector emergente, existe un número reducido de firmas en la región, la mayoría de reciente creación y operando en parte con fondos públicos/privados obtenidos a través de convocatorias, premios e incentivos a la innovación. Todas las firmas encuestadas cuentan con un producto en el mercado.

Luego de identificar los parámetros relevantes, se distinguen dos tipos:

a) Los parámetros del comportamiento que influencian la elección de modelos de acción. En este punto el diseño del cuestionario permite investigar los parámetros: "aceptación del riesgo", "inclinación por la exploración", “aspiración de cuota de mercado" y "aspiración de utilidades".

b) Los parámetros del comportamiento que influencian la elección entre la innovación individual o cooperativa, así como el curso de la cooperación. Estos son la "propensión a cooperar" y la "confianza hacia los socios de cooperación regional".

Dada la importancia de la cooperación innovadora para los SRI, es necesario especificar las condiciones microeconómicas para este tipo de actividad, conectada en el contexto dado del modelo. Las condiciones de activación (triggering conditions) para la cooperación innovadora se derivan de la síntesis del comportamiento y observaciones empíricas (Beckenbach et al., 2009).

Los autores distinguen una combinación de actitudes personales, normas subjetivas y condiciones que influencian la orientación de la voluntad de un agente hacia un modo cooperativo de innovación: en principio, las tres fuerzas que forjan la capacidad de innovar son básicas para la voluntad del agente de cooperar en términos de innovación. Estas fuerzas están relacionadas con el desempeño de mercado actual del agente. En segundo lugar, los diferentes tipos de agentes tienen una propensión diferente a cooperar. Esto toma en consideración que no es sólo importante la posición actual en el mercado, sino también algunas actitudes de mayor profundidad que surgen de diferentes estilos de comunicación dentro de distintos horizontes de innovación. Tercero, por lo menos en un contexto regional, la frecuencia de las innovaciones cooperativas puede ser observada. Esto puede ayudar a reducir la incertidumbre asociada con este tipo de actividad innovadora, al facilitar la búsqueda de socios y demostrar las posibilidades de superar el oportunismo. Cuarto, los subsidios provenientes de instituciones políticas como un incentivo exógeno para formar una cooperación.

Los resultados de Beckenbach et al. (2009) son prácticamente imposibles de replicar con un conjunto de datos pequeño, información no estandarizada y un SRI en formación. Sin embargo, la implementación de la elección de los modelos de acción de las distintas firmas y la variación de su comportamiento a través de las diferentes iteraciones muestra de qué 
manera es posible codificar estas ecuaciones y trasladarlas a un código de programación que permita analizar todo tipo de datos.

En ausencia de métricas, es posible utilizar otras estrategias, como de hecho lo hacen dentro del modelo descrito. Algunas de ellas son la utilización de constantes arbitrarias y/o parámetros definidos de manera aleatoria dentro de ciertos límites.

En el caso de nuestro modelo, se optó por utilizar una variable booleana para identificar si una firma era poseedora o no de conocimiento diferenciado, procediendo a establecer un vínculo de cooperación o match cada vez que la iteración encuentre dos firmas cuya variable contenga el valor 1 , y este vínculo no haya sido establecido previamente.

El código es el siguiente:

set asset-specificity? one-of [false true]

show [asset-specificity?] of turtle 0

show who

show match

if asset-specificity? = true [set potential-match potential-match +1$]$

if counti $=0$ and asset-specificity? = true [set match who

set counti counti +1$]$

if who != match and asset-specificity? = true and potential-match $>1$ [

create-link-with turtle match

set potential-match 0 set counti 0]

fd 1

show count links

El código anterior genera la siguiente salida después de ocho iteraciones, ciclos o ticks, que es la manera en que NetLogo identifica cada vez que culmina un ciclo, en este caso, económico. Este código lo único que hace es generar un estado aleatorio en cada uno de los agentes, que puede ser positivo o negativo. Si es positivo, significa que la firma cuenta con activos específicos (conocimiento, capital); si es negativo, no es candidato a una posible relación de cooperación con otra firma. Así, durante la primera iteración y una vez generados aleatoriamente los primeros estados, se establecen los primeros lazos. Es la forma más sencilla de simular un proceso de cooperación. Sin embargo, carece de elementos de retroalimentación, así como de umbrales y demás variables que caracterizan un proceso de esta naturaleza. 
Figura 4. Salida de resultados en NetLogo de iteraciones 1-8
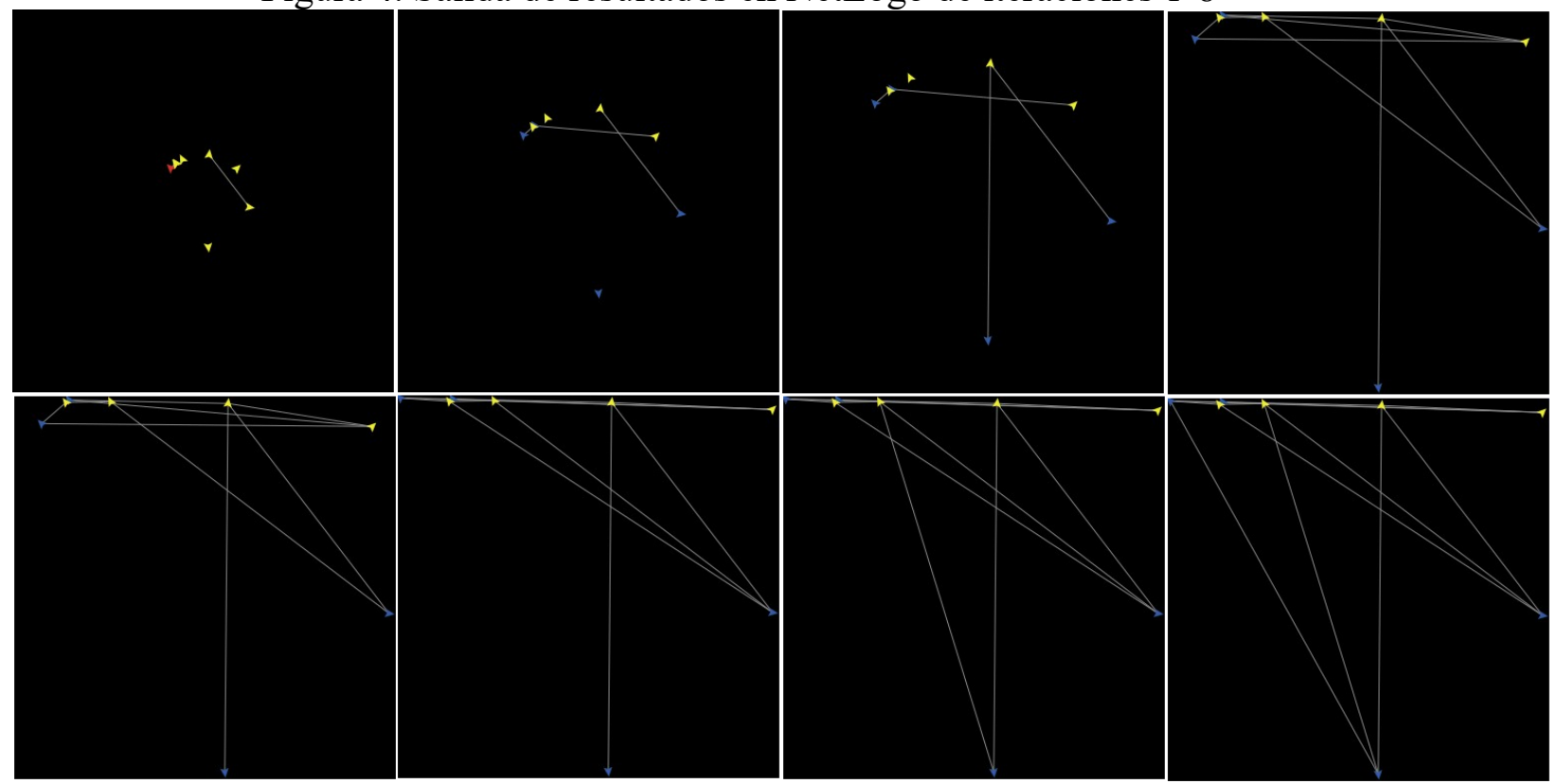

Fuente: Elaboración propia en NetLogo.

En la figura 4 pueden observarse los resultados de correr el modelo durante ocho iteraciones. Así durante el primer ciclo, siete de las ocho firmas asumen el color amarillo, es decir, adoptan un modo de acción innovador cooperativo, y sólo una marca roja, o modo de acción innovador individual. Durante este ciclo se establece solamente una relación de cooperación entre dos firmas. Durante el segundo ciclo, podemos observar una nueva relación de cooperación, y esta vez tres firmas han adoptado el modo rutina (indicado con el color azul). El tercer ciclo marca cuatro relaciones de cooperación. Para el cuarto ciclo, la mitad de las firmas eligen el modo rutina. Durante el octavo ciclo, tenemos 14 lazos, en tanto que la proporción de selección de modo de acción se mantiene constante.

Los resultados observados en la figura anterior reflejan una simplificación respecto a la gran cantidad de variables que intervienen dentro de un proceso de cooperación, desde la consideración de los costos de transacción al verificarse ésta hasta los cambios que deben hacerse en términos algorítmicos para alterar variables tales como la confianza (tr) y la cantidad de conocimiento $(\mathrm{kr})$, que deberán variar con cada cooperación exitosa, o la manera en que dicha cooperación afecta la demanda y/o la cuota de mercado.

Tabla 2. Comportamiento de las variables durante las iteraciones 4 y 8

\begin{tabular}{ccccccccc}
\hline Iteración & Agente & $\mathrm{g}$ & $\mathrm{s}$ & $\mathrm{b}$ & $\mathrm{i}$ & $\mathrm{x}$ & $\mathrm{S} 1$ & $\mathrm{fa}$ \\
\hline \multirow{2}{*}{4} & 0 & 5000000 & 5000000 & 1 & 0 & 1 & 5000000 & 1 \\
& 1 & 1000000 & 1000000 & 1 & 0.1 & 1 & 1000000 & 1 \\
& 2 & 5000000 & 5000000 & 1 & 0.1 & 1 & 5000000 & 1 \\
\hline
\end{tabular}




\begin{tabular}{|c|c|c|c|c|c|c|c|c|}
\hline & 3 & 500000 & 500000 & 1 & 0 & 0.8 & 500000 & 1 \\
\hline & 4 & 1000000 & 1015625 & 1 & 0 & 1 & 1015625 & 0.75 \\
\hline & 5 & 250000 & 252929.6875 & 1 & 0 & 1 & 252929.6875 & 0.75 \\
\hline & 6 & 1000000 & 1005859.375 & 1 & 0 & 1 & 1005859.375 & 0.75 \\
\hline & 7 & 1000000 & 1015625 & 1 & 0 & 1 & 1015625 & 0.75 \\
\hline \multirow[t]{8}{*}{8} & 0 & 5000000 & 5000000 & 1 & 0 & 1 & 5000000 & 1 \\
\hline & 1 & 1000000 & 1000000 & 1 & 0.1 & 1 & 1000000 & 1 \\
\hline & 2 & 5000000 & 5000000 & 1 & 0.1 & 1 & 5000000 & 1 \\
\hline & 3 & 500000 & 500000 & 1 & 0 & 0.8 & 500000 & 1 \\
\hline & 4 & 1000000 & 1000061.0351 & 1 & 0 & 1 & 1000061.0351 & 0.75 \\
\hline & 5 & 250000 & 250011.4440 & 1 & 0 & 1 & 250011.4440 & 0.75 \\
\hline & 6 & 1000000 & 1000022.8881 & 1 & 0 & 1 & 1000022.8881 & 0.75 \\
\hline & 7 & 1000000 & 1000061.0351 & 1 & 0 & 1 & 1000061.0351 & 0.75 \\
\hline
\end{tabular}

Fuente: Elaboración propia con base en los valores obtenidos durante la corrida del modelo en NetLogo.

Conceptualmente, el modelo de Beckenbach et al. (2009) no es más que un proceso iterativo de simulación de cooperación entre los distintos agentes de un sector de un SRI, lo cual implica, entre otras cosas, transferencia de conocimiento y su absorción, así como la generación de productos y/o innovaciones derivadas de dichas relaciones de cooperación. Durante el curso de este proceso iterativo, el inventario de conocimiento de cada firma puede mejorar dependiendo de los distintos factores de retroalimentación del modelo. Conforme avanzan las iteraciones, la confianza puede aumentar o disminuir, dependiendo de si las relaciones de colaboración son exitosas o no.

Los parámetros utilizados para cada una de las variables de los agentes están basados en los datos empíricos recopilados a través de la aplicación del cuestionario de investigación. Debido a la selección muestral, que se centró en firmas dentro del espectro innovador de la biotecnología, la tipología de los agentes es similar, y corresponde a la firma con alto nivel de especificidad de conocimiento y una capacidad de confianza moderada, pero aun así 
dispuesta a la cooperación y a la transferencia de conocimiento, ligada a las firmas orientadas a los servicios, no así a las industriales.

La cantidad de conocimiento transferido durante cada iteración depende del nivel de confianza y la propensión a cooperar de cada firma, y es así como todos estos factores afectan la capacidad general del SRI para producir relaciones de cooperación e innovaciones en el mercado. La generación del código completo de dicha conceptualización es sólo el principio, y deja abierta la puerta a una riquísima mina de exploración, que permitirá probar la influencia de distintas variables en la eficiencia de un SRI.

Desafortunadamente, dichas consideraciones rebasan los alcances de este texto. Sin embargo, y aunque se espera abordarlas extensivamente en un futuro, consideramos que el código generado es suficiente para ilustrar la poderosa herramienta a la que tiene acceso la ciencia social al incorporar la investigación basada en SAC, que permite modelar todo tipo de sistemas con la ayuda de un lenguaje de programación relativamente sencillo y ampliamente utilizado por la comunidad científica.

\section{CONCLUSIONES Y RECOMENDACIONES}

En América Latina falta mucho camino por recorrer en términos de homogenización de datos respecto a la medición de la innovación. En Sonora, son todavía poquísimas las compañías dedicadas a la biotecnología, y aún menos las dispuestas a proporcionar información, por lo que el análisis de datos disponibles es un ejercicio meramente exploratorio.

La mayor parte de las compañías exploradas se autoidentifica como innovadora, tendiente a la innovación cooperativa, dueña de capital humano altamente especializado, poseedora de un alto nivel de conocimiento $\mathrm{y}$, en general, se traza metas relativamente ambiciosas en términos de utilidades, penetración y ampliación del mercado.

Lo anterior desentona con algunas observaciones hechas en el transcurso de esta investigación, como la marcada reticencia a la cooperación mostrada en la respuesta a un cuestionario entre un amplio grupo de agentes, incluyendo académicos y empresarios, y cierta desconfianza por parte de actores gubernamentales. Estos últimos debieron excluirse del conjunto de datos de este modelo, por lo que se recomienda una futura investigación que los incorpore.

El potencial está latente; compañías como Rubio Pharma, Agropro y Galaz Science and Engineering, dedicadas respectivamente a la farmacología, la agricultura de precisión y la fabricación de instrumentos biomédicos, están fuertemente conectadas hacia el exterior, y tienen lazos significativos con centros de investigación e instituciones centralizadas, IES locales y universidades extranjeras.

La comunidad científica debe permanecer alerta ante la evolución del comportamiento del SRI, estudiando este y otros sectores emergentes, alimentando los conjuntos de datos 
disponibles y proveyendo información económica relevante para, en la medida de lo posible, ser agente de cambio en su crecimiento y consolidación.

En el modelo de simulación implementado por Beckenbach et al. (2009) en Alemania, la innovación cooperativa juega un importante rol en la derrama de conocimiento a nivel regional. Los comportamientos diferenciados de los agentes no sólo les otorgan cadenas de conocimiento heterogéneas, sino que el conocimiento para la región como un todo es diferente en cada ciclo, y la importancia de cada rama del conocimiento varía durante estos.

En general, para Beckenbach et al. (2009), el número de innovaciones cooperativas y la derrama de conocimiento entre agentes son los indicadores más importantes del desempeño del SRI. Los agentes que eligen el modo de innovación cooperativa son el core de este, y este modo de acción es la fuente de redes de relaciones multiplex tanto de mercado como de jerarquía. Este análisis muestra que los parámetros para la innovación cooperativa revelan un "paisaje de red" detrás de las dinámicas observables en un SRI.

La ampliación de la perspectiva hacia este paisaje ilumina las condiciones para un buen desempeño. Dichas simulaciones, por tanto, pueden ser un punto de partida para la mejora bottom up, o de abajo hacia arriba, de los SRI -incluidas las redes-, en contraste con la perspectiva usual de optimización top-down en investigación de redes, y la correspondiente entre los "diseñadores" o "master minds" detrás de un SRI.

Investigaciones futuras de esta índole en Sonora y en otras regiones del mundo con SRI en proceso de formación, requerirán de la incorporación de nuevas variables para el estudio prospectivo de los distintos sectores productivos y de las firmas que los componen, especialmente aquéllas relacionadas con la cooperación y colaboración científica y tecnológica entre éstas, además de asegurarse de su claridad técnica y replicabilidad.

Asimismo, queda manifiesta la urgente necesidad de colaboración de la ciencia social con las ciencias informáticas y computacionales, necesarias como puente para la generación de simulaciones multiagente basadas en teoría social y económica llevada al plano algorítimico.

\section{REFERENCIAS}

Asheim, B. T. y Isaksen, A. (2002). Regional Innovation Systems: The Integration of Local 'Sticky' and Global 'Ubiquitous' Knowledge. The Journal of Technology Transfer, 27(1), 77-86. https://doi.org/10.1023/A:1013100704794

Beckenbach, F., Briegel, R. y Daskalakis, M. (2007). Behavioral foundation and agent-based simulation of regional innovation dynamics. Papers on Agent Based Economics No. 3. Alemania: University of Kassel-Institute of Economics.

Beckenbach, F., Briegel, R. y Daskalakis, M. (2009). Evolution and Dynamics of Networks in 'Regional Innovation Systems' (RIS). En A. Pyka y A. Scharnhorst (Eds.), Innovation Networks: New Approaches in Modelling and Analyzing, (pp. 59-100). Suiza: Springer/Verla. https://doi.org/10.1007/978-3-540-92267-4_4 
Carrillo, J. y Contreras, Ó. F. (2015). Experiencias estatales y transfronterizas de innovación en México. Tijuana: El Colegio de la Frontera Norte.

Cooke, P. (2008). Regional innovation systems: origin of the species. International Journal of Technological Learning, Innovation and Development, 1(3), 393-409.

Daskalakis, M. (2016). Behavioral Determinants of Environmental Innovation: A Carnegie based approach. En F. Beckenbach y W. Khalenborn (Eds.), New Perspectives for Environmental Policies Through Behavioral Economics, (pp. 301-323). Suiza: Springer Books. https://doi.org/10.1007/978-3-319-16793-0

Giuliani, E. y Pietrobelli, C. (2011). Social Network Analysis Methodologies for the Evaluation of Cluster Development Programs. En A. Maffioli, C. Pietrobelli y R. Stucchi (Eds.), The Impact Evaluation of Cluster Development Programs, (pp. 37-58). Washington, DC: Interamerican Development Bank (IDB).

Hein, O., Schwind, M. y König, W. (2006). Scale-free networks. WIRTSCHAFTSINFORMATIK, 48(4), 267-275. https://doi.org/10.1007/s11576-006$\underline{0058-2}$

Holland, J. H. (2006). Studying Complex Adaptive Systems. Journal of Systems Science and Complexity, 19(1), 1-8. https://doi.org/10.1007/s11424-006-0001-z

Kafarski, P. (2012). Rainbow code of biotechnology. CHEMIK, (4), 811-816.

Matyushenko, I., Sviatukha, I. y Grigorova-Berenda, L. (2016). Modern Approaches to Classification of Biotechnology as a Part of NBIC-Technologies for Bioeconomy. British Journal of Economics, Management \& Trade, 14(4), 1-14. https://doi.org/10.9734/BJEMT/2016/28151

Molle, W. y Djarova, J. (Eds.). (2009). Enhancing the Effectiveness of Innovation: New Roles for Key Players. Reino Unido/Estados Unidos: Edward Elgar Pub.

Snodgrass, R. T. (2011). An interview with Melanie Mitchell: On complexity. Ubiquity, 2011, 1-6. https://doi.org/10.1145/1967045.1967047

Taylor, M. C. (2003). The Moment of Complexity. Emerging Network Culture. Estados Unidos: University of Chicago Press. Recuperado de https://www.press.uchicago.edu/ucp/books/book/chicago/M/bo3615087.html 